\title{
A NOTE ON MULTIVARIATE POISSON FLOWS ON STOCHASTIC PROCESSES
}

\author{
FREDERICK J. BEUTLER, ${ }^{*}$ The University of Michigan
}

\begin{abstract}
In [1], a deterministic counting rate condition is shown to be necessary and sufficient for a counting process induced on a Markov step process $Z$ to be multivariate Poisson. We show here that the result continues to hold without $Z$ being a Markov step process.
\end{abstract}

MARKOV STEP PROCESS

It was assumed in [1] that a Markov step process $Z$ induces a multivariate counting process $N=\left(N_{1}, N_{2}, \cdots, N_{c}\right)$. The infinitesimal generator $A$ of $Z$ was used there to characterize a vector process whose respective components $r_{i}(Z(t))$ can be heuristically interpreted as the counting rates for the corresponding $N_{i}$ at time $t$. It is shown in [1] that if the components of $N$ do not have simultaneous jumps, a determinacy condition based on the sigma algebras $N_{t}=\sigma\{N(u), u \leqq t\}$ is necessary and sufficient for $N$ to consist of mutually independent Poisson processes. This condition is that for each $t$ we have almost surely

$$
E\left[r(Z(t)) \mid \mathbf{N}_{t}\right]=E[r(Z(t))]
$$

The above result is extended in the present letter to processes $Z$ that need not be Markov. To this end, let $Z$ be measurable with respect to an increasing family of sigma algebras $\left\{\boldsymbol{F}_{t}\right\}$, and suppose further that $Z$ induces the counting process $N$ (as defined in [2], Chapter 2) in the sense that $\boldsymbol{N}_{t} \subseteq \boldsymbol{F}_{t}$ for each $t$. Let $E\left[N_{i}(t)\right]<\infty$ for each $t$, $i=1,2, \cdots, c$, with the $N_{i}$ having the respective $\boldsymbol{F}_{t}$-intensities (see [2], II.D7) $\lambda_{i}$. It is also presumed that the conditional expectations $E\left[\lambda_{i}(\cdot) \mid \mathbf{N}\right]$ have an $\mathbf{N}_{t}$-progressive version, which we can (and shall) assume to be $\boldsymbol{N}_{t}$-predictable ([2], Theorem II.T13) without loss of generality.

Now if $I$ stands for the indicator function, it is tautologically true that

$$
I\left[N_{i}(t)-N_{i}(s)>n_{i}\right]=\int_{s}^{t} I\left[N_{i}(u-)-N_{i}(s)=n_{i}\right] d N_{i}(u)
$$

for any $0 \leqq s \leqq t$. (Equation (2), together with its possible implications, were called to the author's attention by Dr B. Melamed.) Moreover, $N_{i}(t)-\int_{0}^{t} \lambda_{i}(s) d s$ is not only an $\boldsymbol{F}_{\mathrm{t}}$-martingale, but also a fortiori an $\mathbf{N}_{\mathrm{t}}$-martingale. It then follows from the definition of

\footnotetext{
Received 26 October 1982.

* Postal address: Computer, Information and Control Engineering Program, The University of Michigan, Ann Arbor, MI 48109, U.S.A.
} 
intensity that on the right side of (2)

(3) $E\left[\int_{s}^{t}\left[N_{i}(u-)-N_{i}(s)=n_{i}\right] d N_{i}(u) \mid N_{s}\right]=E\left[\int_{s}^{t}\left[N_{i}(u)-N_{i}(s)=n_{i}\right] \lambda_{i}(u) d u \mid N_{s}\right]$.

Equations (2) and (3) may be combined by taking the conditional expectation in (2) respective to $\boldsymbol{N}_{s}$, and substituting. If we then also add over $n_{i}=0,1,2, \cdots$ and apply Fubini's theorem, we obtain

$$
E\left[N_{i}(t)-N_{i}(s) \mid \mathbf{N}_{s}\right]=\int_{s}^{t} E\left[\lambda_{i}(u) \mid \mathbf{N}_{s}\right] d u .
$$

This equation effectively generalizes (1.18) of [1]; our $\lambda_{i}$ plays the role of the $r_{i}$ of [1], which in [1] is generated by a Markov step process $Z$. Indeed, under the assumptions of [1], our (4) specializes precisely to Equation (1.18) in [1].

Condition (3.2) in [1] may be replaced by

$$
E\left[\lambda_{i}(t) \mid \mathbf{N}_{t}\right]=E\left[\lambda_{i}(t)\right]
$$

almost surely with respect to $d t d P$ measure. As in [1], this condition (in the presence of the preceding hypotheses on $N, \boldsymbol{N}_{t}, \boldsymbol{F}_{t}$, and $E\left[\lambda_{i}(\cdot) \mid \boldsymbol{N}\right]$ above) is necessary and sufficient for $N$ to be a multivariate Poisson process respective to $\boldsymbol{N}_{t}$. The proofs are easy exercises in the martingale theory of multivariate counting processes.

If (5) is met, we have in (4)

$$
E\left[\lambda_{i}(u) \mid \mathbf{N}_{s}\right]=E\left\{E\left[\lambda_{i}(t) \mid \mathbf{N}_{t}\right] \mid \mathbf{N}_{s}\right\}=E\left[\lambda_{i}(t)\right]
$$

Thus $N$ is a multivariate Poisson process according to the multichannel Watanabe theorem (see [2], Theorem II.T6). Conversely, let $N$ be multivariate Poisson. From (4) and the $\boldsymbol{N}_{t}$-independent increment property it follows that $N_{i}$ has the predictable $\mathbf{N}_{t}$-intensity $E\left[\lambda_{i}(\cdot)\right]$. But also, a version of $E\left[\lambda_{i}(\cdot) \mid \mathbf{N}\right]$ is such an intensity (see [2], Theorem II.T14). The uniqueness of predictable intensities ([2], Theorem II.T12) then yields (5), as was desired.

\section{References}

[1] Beutler, F. J. And Melamed, B. (1982) Multivariate Poisson flows on Markov step processes. J. Appl. Prob. 19, 289-300.

[2] Brémaud, P. (1981) Point Processes and Queues: Martingale Dynamics. Springer-Verlag, New York. 